\title{
Quantification of Spice Mixture Compositions by Electronic Nose: Part I. Experimental Design and Data Analysis Using Neural Networks
}

\author{
HAOXIAN ZHANG, MURAT Ö. BALABAN, JOSÉ C. PRINCIPE, AND KENNETH PORTIER
}

\begin{abstract}
A quantitative procedure was developed to predict the composition of ternary ground spice mixtures using an electronic nose. Basil, cinnamon, and garlic were mixed in different compositions and presented to an enose. Nineteen training mixtures were used to build predictive models. Model performance was tested using 5 other mixtures. Three neural network structures-multilayer perceptron (MLP), MLP using principal component analysis as a preprocessor (PCA-MLP), and the time-delay neural network (TDNN)-were used for predictive model building. All 3 neural network models predicted the testing mixtures' compositions with a mean square error (MSE) equal or less than 0.0051 (in a fraction domain where sum of fractions $=1$ ). The TDNN provided the smallest MSE.

Keywords: electronic nose, mixture, neural network, quantification, spice
\end{abstract}

\section{Introduction}

$\mathrm{E}$ lectronic noses (e-noses) consisting of olfactory sensors and multivariate signal analysis methods can quickly detect and distinguish odors at low cost. Currently, the biggest application of e-noses is in food-related areas (Gardner and Bartlett 1999; Zhang 2003b).

E-nose studies have generally focused on investigating qualitative aspects of samples, for example, detecting or classifying odor patterns, such as "fresh" versus "spoiled" (Harper 2001). The traditional data analysis methods for e-noses were designed for pattern classification or recognition. Even in the few studies dealing with quantitative attributes of samples (Alpha M.O.S. 1997; Dittmann and others 2000), the samples with different ingredient percentages were treated as samples with different qualitative attributes (for example, patterns) during data analysis.

No work was found in applying e-noses to quantitatively predict ingredient percentages. In the food and fragrance industries, many products are formed by mixing 2 or more ingredients with different olfactory attributes. A simple, fast ingredient percentage prediction tool for such products would be of great value for routine quality or process control.

The ingredient percentages prediction is harder than classification and requires extra care in the experimental design and the definition and training of the neural networks. In mixture experiments, the sum of all fractions must add up to 1 , and the fraction of each component must be non-negative. Networks have to be trained with desired responses that are real numbers instead of the common $0 / 1$ in pattern classification. Mixture prediction is therefore a regression instead of a classification problem.

The objectives of this study are to develop an experimental procedure that can quantify ingredient percentages using an e-nose

MS 20040331 Submitted 5/19/04, Revised 7/18/04, Accepted 1/10/05. Author Zhang is with Agricultural and Biological Engineering Dept., Univ. of Florida, Gainesville, Fla. Author Balaban is with Food Science and Human Nutrition Dept., Univ. of Florida, P.O. Box 110370, Gainesville, FL 32611. Author Principe is with Computational NeuroEngineering Laboratory, Univ. of Florida, Gainesville, Fla. Author Portier is with Department of Statistics, Univ. of Florida, Gainesville, Fla. Direct inquiries to author Balaban (E-mail: MOBalaban@mail.ifas.ufl.edu). as the measurement tool and to evaluate artificial neural network topologies for nonlinear regression.

A multilayer perceptron (MLP) trained by back-propagation, a simple and popular neural network topology, can be a method for quantitative data analysis (Haykin 1999; Principe and others 1999). A time-delay neural network (TDNN), a dynamic network with embedded local memory, has been shown to improve an enose's pattern recognition ability (Zhang and others 2003). TDNNs are able to exploit the temporal information of the e-nose responses unlike any of the other models. Therefore, TDNN was selected as another possible topology, with a potential to perform better than MLP. Other data analysis methods include incorporating principal component analysis (PCA) (Principe and others 1999; Khattree and Naik 2000), a multivariate statistical method that can preserve information in a less dimensional format, with the neural network structures described previously.

Spice mixtures were selected as the model system in this study because spices have high volatiles content easily detectable by an e-nose; they are stable during storage, making a large experiment feasible; they are valuable for their olfactory attributes; and they are regularly used as mixtures in the industry.

For odor analysis, there are 2 traditional competitors of e-noses: gas chromatography (GC) and sensory analysis. The assessment of how well a newly developed e-nose procedure performs in predicting a mixture composition should be based on the comparison of the performance between the e-nose and the 2 traditional methods. The performance of the 2 traditional methods on the selected mixtures and their comparison to e-nose performance were investigated in part II of this study.

\section{Materials and Methods}

\section{Spices}

Three ground spices-basil (Ocimum basilicum), cinnamon (Cinnamomum Cassia), and garlic (Allium sativum)—were selected to form the spice mixtures. This selection was based on the following criteria: (1) the major volatile components of the 3 spices should be different from each other and should be easily separated by 
Table 1-Common and formal chemical names, retention index (RI) for a DB-5 column, and main odor descriptions of major volatile compounds of basil, cinnamon, and garlic

\begin{tabular}{|c|c|c|c|c|}
\hline Spice & Common name & Formal name & RI (DB5) & Odor \\
\hline Basil & Linalool & 1,6-octadien-3-ol, 3,7-dimethyl- & 1100 & Flower, lavender \\
\hline Basil & Estragole & Benzene, 1-methoxy-4-(2-propenyl)- & 1195 & Licorice, anise \\
\hline Basil & Eugenol & Phenol, 2-methoxy-4-(2-allylphenol)- & 1356 & Clove \\
\hline Basil & 1,8-cineole & 1,8-epoxy-p-menthane & 1030 & Mint, sweet \\
\hline Cinnamon & trans-Cinnamaldehyde & 2-propenal, 3-phenyl, (E)- & 1266 & Cinnamon \\
\hline Cinnamon & Cinnamyl acetate & 2-propen-1-ol, 3-phenyl-, acetate & 1443 & $\mathrm{~N} / \mathrm{A}$ \\
\hline Cinnamon & No:trans-Cinnamic acid & trans-3-phenyl-2-propenonic acid & 1438 & Honey \\
\hline Cinnamon & Benzaldehyde & Benzaldehyde & 968 & Almond, burnt sugar \\
\hline Garlic & Diallyl disulfide & N/A & 1320 & Garlic \\
\hline Garlic & Diallyl sulfide & $\mathrm{N} / \mathrm{A}$ & 1085 & N/A \\
\hline Garlic & Methyl allyl disulfide & $\mathrm{N} / \mathrm{A}$ & 1149 & $\mathrm{~N} / \mathrm{A}$ \\
\hline
\end{tabular}

GC, thus facilitating the mixture analysis by GC; (2) the odors of the 3 spices should be significantly different from each other and should be pleasant to smell, which facilitates the sensory analysis using human subjects. As shown in Table 1, the major volatile components of basil, cinnamon, and garlic were different. Their Kovats retention indices (RI) were also different enough to allow acceptable separation (Adams 1995; Uhl 2000; Garbe and others 2001; Acree and Arn 2004). In a preliminary screening study, 8 human subjects (all graduate student from the Food Science Dept. in the Univ. of Florida, with no self-reported problems in their sense of smell) were presented with the spice samples, which have been prepared to avoid giving any visual clues to the subject about the compositions of the spice samples (Zhang and others 2005). The subjects were asked to identify each of the 3 spices they smelled and to rate their confidence in identification as "low," "medium," or "high." The subjects were also asked to rate the hedonic attribute ("unpleasant," "neutral," or "pleasant") of the smell of each spice and its mixture (in a mix ratio of 1:1:1). All 8 subjects correctly identified the 3 spices and rated their confidence of identification as high. The hedonic ratings toward the pure spice and spice mixture

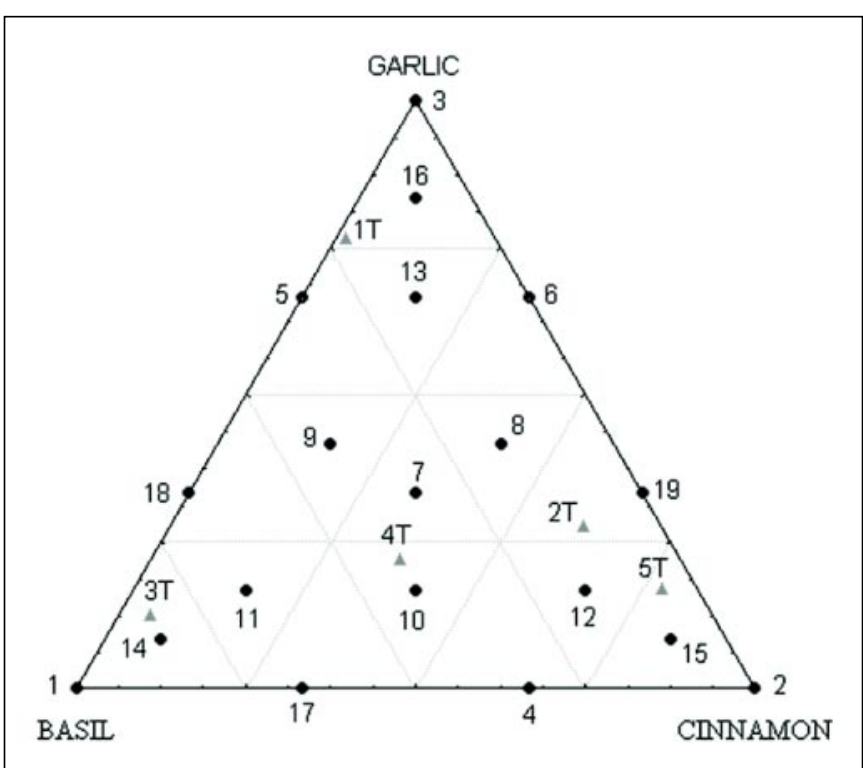

Figure 1-The distribution of design points in the simplex space. The dots, numbered from 1 to 19 , represent the spice mixtures used for training/cross-validation, and the small triangles, denoted as $1 \mathrm{~T}$ through $5 \mathrm{~T}$, represent those for testing. were either "pleasant" or "neutral." The results with human subjects showed that the 3 spices smelled significantly different and had pleasant odors whether in pure form or in the formulated mixtures.

Basil was purchased from Cibolo Junction Food \& Spice Co. (Albuquerque, N.Mex., U.S.A.). Cinnamon and garlic were purchased from Frontier Natural Products (Norway, Iowa, U.S.A.). The 3 spices were individually equilibrated with a saturated potassium carbonate solution at $20{ }^{\circ} \mathrm{C}$ for $20 \mathrm{~h}$ to reach the same relative humidity (43.2\%). Therefore, the effects of different humidities on e-nose sensors were eliminated.

\section{Experimental design}

To obtain sufficient data for prediction model building, the training points should have a satisfactory distribution throughout the experimental region and provide an internal estimate of the error variance (Cornell 1990). Furthermore, the points that test the performance of the model built should be randomly distributed in the experimental region. The selected spice mixtures for training/weight fraction of basil, cinnamon, and garlic are represented by $x_{1}, x_{2}$, and $x_{3}$, respectively. In the testing spice blends, the fractions $x_{1}, x_{2}$, and $x_{3}$ were generated using the random function in Microsoft Excel: $x_{1}=R A N D(), x_{2}=R A N D()^{*}\left(1-x_{1}\right)$, and $x_{3}=1-x_{1}-x_{2}$. Eight samples were prepared for each training/crossvalidation point, and 5 samples for each testing point.

Spice mixtures were formulated in the weight fractions listed in Table 2. Each experimental sample contained a 10-g formulated spice mixture. The prepared samples were then presented to an e-nose (e-Nose 4000, Neotronics, Gainesville, Ga., U.S.A.) with 12 conducting polymer sensors (sensor types: 483, 478, 464, 463, 462, $461,460,459,458,401,298$, and 297). The order of sample presentation was randomized. For each measurement, the sample chamber was purged by compressed dry air (BOC GASES, Murray Hill, N.J., U.S.A.) for $4 \mathrm{~min}$ and the sensor chamber was purged $2 \mathrm{~min}$ to eliminate foreign/residual odors. The flow rate of the compressed air used for both chambers was $800 \mathrm{~cm}^{3} / \mathrm{min}$. The sensors' responses were recorded every second up to $4 \mathrm{~min}$. The experiments were run at room temperature $\left(23^{\circ} \mathrm{C}\right.$ to $\left.25^{\circ} \mathrm{C}\right)$.

\section{Data analysis}

Three neural network methods were used to analyze data obtained from the e-nose experiments: MLP, PCA-MLP, and TDNN, described subsequently in detail.

MLP. Twelve inputs and 3 outputs were used in the MLP. Each input corresponded to 1 of the 12 e-nose sensors' instantaneous response at $4 \mathrm{~min}$. Each of the 3 outputs indicated the mass fraction of a spice (basil, cinnamon, or garlic) in the mixture. The activation function of the output layer was a softmax, which restricts each out- 
Table 2-The weight fractions of design points for training and testing ${ }^{a}$

\begin{tabular}{cccc}
\hline Mix $\mathbf{n r}$ & $\boldsymbol{x}_{\mathbf{1}}$ (basil) & $\boldsymbol{x}_{\mathbf{2}}$ (cinnamon) & $\boldsymbol{x}_{\mathbf{3}}$ (garlic) \\
\hline 1 & 1.0 & 0.0 & 0.0 \\
2 & 0.0 & 1.0 & 0.0 \\
3 & 0.0 & 0.0 & 1.0 \\
4 & 0.333 & 0.667 & 0 \\
5 & 0.333 & 0.0 & 0.667 \\
6 & 0 & 0.333 & 0.667 \\
7 & 0.333 & 0.333 & 0.333 \\
8 & 0.167 & 0.417 & 0.417 \\
9 & 0.417 & 0.167 & 0.417 \\
10 & 0.417 & 0.417 & 0.167 \\
11 & 0.667 & 0.167 & 0.167 \\
12 & 0.167 & 0.667 & 0.167 \\
13 & 0.167 & 0.167 & 0.667 \\
14 & 0.833 & 0.083 & 0.083 \\
15 & 0.083 & 0.833 & 0.083 \\
16 & 0.083 & 0.083 & 0.833 \\
17 & 0.667 & 0.333 & 0 \\
18 & 0.667 & 0.0 & 0.333 \\
19 & 0 & 0.667 & 0.333 \\
$1 \mathrm{~T}$ & 0.218 & 0.015 & 0.767 \\
$2 \mathrm{~T}$ & 0.114 & 0.610 & 0.277 \\
$3 \mathrm{~T}$ & 0.829 & 0.047 & 0.124 \\
$4 \mathrm{~T}$ & 0.413 & 0.366 & 0.221 \\
$5 \mathrm{~T}$ & 0.052 & 0.777 & 0.171 \\
\hline
\end{tabular}

aMixtures 1 through 19 were used for training, whereas mixtures 1 through 5T were used for testing.

put to a value between 0 and 1 and makes them sum up to 1 (Principe and others 1999). By using softmax activation function in the output layer, the internal constraint of the mixture experiments (the mass fractions of 3 spices must add up to 1) was satisfied. One hidden-layer was selected for the MLP because this topology provides universal mapping capabilities (Principe and others 1999). In our case, sensors' responses toward mixtures were expected to be continuous. The other requirement for model estimation is the desired outputs during training. In classification, the desired responses are 0 or 1 , but here the desired responses have to be fractional values because the goal is to predict the percentage of each ingredient in the mixture. Therefore, we use the values in Table 2, which are the experimental fractions of the corresponding spice mixtures as the desired outputs, when training the neural networks. Training was stopped with cross-validation to maximize the generalization performance of the trained networks. The training/cross-validation data set was partitioned into a training set and a cross-validation set: 2 replicates from each spice mixture were randomly selected to build the cross-validation set, and the remaining 6 replicates were used as the training set. The order of replicates in the training set was randomized before presenting to a neural network to avoid training bias.

The selection of the suitable MLP topology (the number of neurons in the hidden layer) and parameters (weights and biases) was based on the performance of the topology/parameters in the testing data. The selection of topology/parameters proceeded as follows: First, a network topology was selected. This network was trained at least 10 times with random initial conditions based on how variable the testing results were, and the best testing result was recorded for that topology. Then, the network topology was changed, and the same training method was applied. This iterative method was continued until performance on testing data could not be improved. By the above procedure, the optimal topology-parameter combination of MLP was determined, and the corresponding testing result was recorded as the performance of the network.

In this study, the testing result of the neural network topology-pa- rameters combination was obtained by averaging the 5 replicates of enose's responses corresponding to each testing point and then using these average responses as the inputs of that neural network. The error of a testing result was measured by mean square error (MSE).

PCA-MLP. This method was similar to that of MLP except that PCA was 1st applied to instantaneous sensor responses to reduce dimensionality of the original data. After the principal components that explain less variance were eliminated, the PCA resulted in a reduced number of inputs into the neural network. Then, PCA scores instead of original sensor responses were used as inputs to the neural network. The number of inputs was equal to the number of principal components selected; therefore, the advantage of this method is that it reduces the overall number of network parameters. However, there is no guarantee that the principal component subspace chosen will provide discriminability.

TDNN. The TDNN method was similar to MLP in its number of inputs, outputs, and hidden layers used in the network, its use of the training, cross-validation and testing data sets, and its ways to select suitable network topology. The optimal weights and biases can still be automatically found with the back-propagation algorithm used in MLP method. The TDNN method was different from that of MLP in the aspects described below.

First, the inputs used in the TDNN were different. The entire time series sensor responses from 0 to $4 \mathrm{~min}$ ( $1 \mathrm{reading} / \mathrm{s}$ ) instead of the instantaneous sensor responses at $4 \mathrm{~min}$ were used (240 readings). Second, embedded local memories (tapped delay line) in both input and hidden layers of the TDNN made it sensitive to the sequence of input values. Third, the outputs of the TDNN were also time series data. The 3 desired outputs (the mass fractions of basil, cinnamon, and garlic) were set as the experimental fractions of the corresponding spice in the mixture, regardless of the time at which the sensor responses were collected. The predicted mixture compositions for each spice were based on the average of the corresponding outputs over time.

The data set partitioning into training and cross-validation sets, and ordering (randomization) were accomplished in Microsoft Excel spreadsheets using either the random/sort function or customized Visual Basic for Applications (VBA) codes. PCA was performed using SAS $^{\circledR}$ (SAS Inst. Inc., Cary, N.C., U.S.A.), and neural networks were implemented using NeuroSolutions ${ }^{\circledR}$ (NeuroDimension Inc., Gainesville, Fla., U.S.A.).

\section{Results and Discussion}

$\mathrm{T}$ he optimal topology of MLP was determined to be 1 hidden lay er with 12 inputs/ 3 outputs and 4 hidden neurons (Figure 2). The activation function of the hidden layer was sigmoid, and that of output layer was softmax. This optimal MLP predicted the mixture compositions with an error of 0.0051 (MSE). Table 3 lists the prediction values for each spice mixture at each testing point.

It is important for the PCA-MLP method to select suitable principal component scores as the inputs to a neural network. The selection of principal components is generally based on the amount of variance that each principal component preserves; however, the optimal number to preserve discriminability is data-dependent. It was experimentally found that the 1 st 2 principal components provided as good results in the training set as higher subgroups of the principal components. Based on this, only the 1st 2 principal component scores were selected as neural network inputs because they result in the smallest number of parameters in the network, which provides the best generalization.

The optimal topology of PCA-MLP was determined to be 2 inputs/ 3 outputs and 1 hidden layer with 4 hidden neurons (Figure 3 ). The prediction values for each spice mixture at each testing point are list- 
Table 3-The experimental and predicted mass fractions of spice mixtures predicted by the optimal multilayer perceptron (MLP)

\begin{tabular}{|c|c|c|c|c|c|c|c|}
\hline \multirow[b]{2}{*}{ Testing points } & \multicolumn{3}{|c|}{ Experimental weight fraction } & \multicolumn{3}{|c|}{ Predicted weight fraction } & \multirow{2}{*}{$\begin{array}{l}\text { Average absolute } \\
\text { prediction error }\end{array}$} \\
\hline & Basil & Cinnamon & Garlic & Basil & Cinnamon & Garlic & \\
\hline $1 \mathrm{~T}$ & 0.218 & 0.015 & 0.767 & 0.226 & 0.099 & 0.675 & 0.061 \\
\hline $4 \mathrm{~T}$ & 0.413 & 0.366 & 0.221 & 0.349 & 0.407 & 0.244 & 0.043 \\
\hline \multirow[t]{2}{*}{$5 \mathrm{~T}$} & 0.052 & 0.777 & 0.171 & 0.049 & 0.926 & 0.024 & 0.100 \\
\hline & & & & \multicolumn{4}{|c|}{ Mean Square Error $(\mathrm{MSE})=0.0051$} \\
\hline
\end{tabular}

Table 4-The experimental and predicted mass fractions of spice mixtures predicted by the optimal multilayer perceptron (MLP) using principal component analysis as a preprocessor (PCA-MLP)

\begin{tabular}{cccccccc}
\hline & \multicolumn{2}{c}{ Experimental weight fraction } & \multicolumn{2}{c}{ Predicted weight fraction } & $\begin{array}{c}\text { Average absolute } \\
\text { prediction error }\end{array}$ \\
\cline { 2 - 7 } Testing points & Basil & Cinnamon & Garlic & Basil & Cinnamon & Garlic & 0.037 \\
1T & 0.218 & 0.015 & 0.767 & 0.162 & 0.05 & 0.787 & 0.291 \\
2T & 0.114 & 0.61 & 0.277 & 0.08 & 0.629 & 0.124 & 0.008 \\
3T & 0.829 & 0.047 & 0.124 & 0.841 & 0.035 & 0.222 & 0.089 \\
4T & 0.413 & 0.366 & 0.221 & 0.28 & 0.498 & 0.061 \\
5T & 0.052 & 0.777 & 0.171 & 0.04 & 0.869 & Mean Square Error (MSE) = 0.0041 \\
\hline
\end{tabular}

ed in Table 4. The activation function of the hidden layer was sigmoid and that of output layer was softmax. This optimal topology predicted the mixture compositions with an error of 0.0041 (MSE).

It was experimentally found that the TDNN's performance (measured by MSE resulting from the testing set) was improved by averaging every 4 seconds' responses of e-nose sensors. The averaging procedure removed part of the noise from the time series data. The best TDNN performance was obtained by using the time series sensors' responses collected between 2 and $4 \mathrm{~min}$. This indicated that the time-related information accounting for discrimination was more abundant in the later portion of the e-nose time series data, from 2 to $4 \mathrm{~min}$. Because the TDNN for the entire e-nose response would require longer delay lines and more weights, it could decrease performance.

For a TDNN topology, the optimal weights and biases are searched by a back-propagation algorithm. There were several other independent parameters to be chosen by the user: the number of neurons in the hidden layer, the depth (number of taps) and

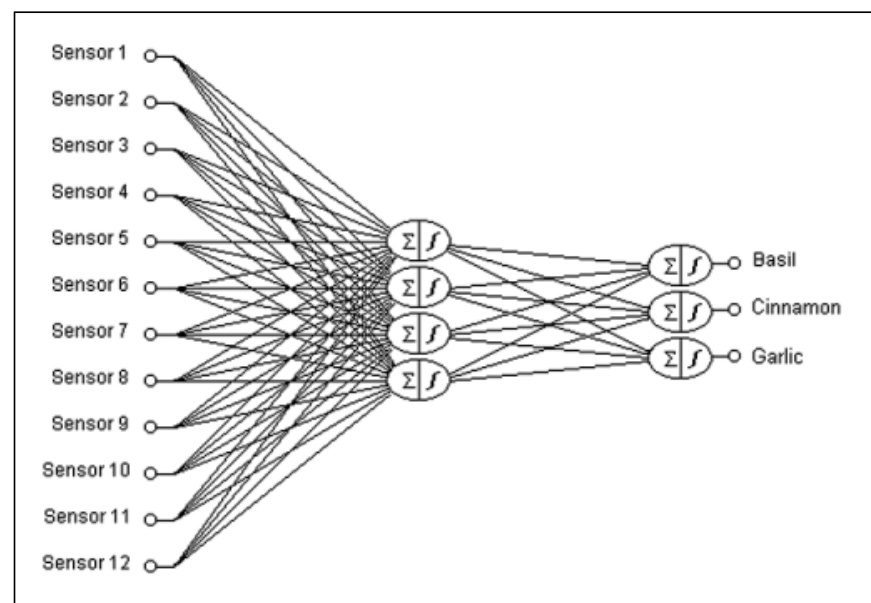

Figure 2-The optimal topology of multilayer perceptron (MLP) resolution (each tap delays the signal by a certain number of time units) of each memory structure. To search for the best combination of the above parameters, only 1 of these parameters was adjusted at a time until the best performance was found. It was found that the best TDNN performance predicted the mixture compositions with an error of 0.0035 (MSE). The prediction values for each spice mixture at each testing point are listed in Table 5. The optimal architecture of TDNN, as shown in Figure 4, is described as follows:

12 inputs/ 3 outputs and 1 hidden layer with 10 neurons;

The activation function of the hidden layer was tanh.

The activation function of the output layer was softmax.

The number of taps in the input layer was 6, with 1 tap delay.

The number of taps in hidden layer was 3, with 1 tap delay.

The prediction MSE values showed that in this study, PCA-MLP performed marginally better than MLP, and TDNN performed marginally better than PCA-MLP in predicting mixture compositions. Just like any other modeling structure, the generalization ability of an MLP model will deteriorate with increasing number of parameters, given the same amount of input/output information (Haykin 1999). PCA extracted most of the inputs' variance (over $99 \%$ ) in a reduced dimensional format (from 12 to 2 dimensions). Because the number of parameters necessary in a MLP structure was directly correlated with the dimensions of its inputs, the preprocessing of input information by PCA significantly decreased the number of parameters necessary in the MLP structure. That resulted in improved generalization ability and a better performance of

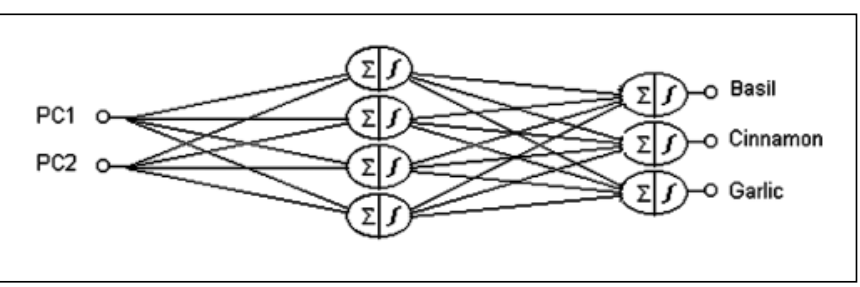

Figure 3-The optimal topology of multilayer perceptron (MLP) using principal component analysis as a preprocessor (PCA-MLP) 
Table 5-The experimental and predicted mass fractions of spice mixtures predicted by the optimal time-delay neural network (TDNN)

\begin{tabular}{|c|c|c|c|c|c|c|c|}
\hline \multirow[b]{2}{*}{ Testing points } & \multicolumn{3}{|c|}{ Experimental weight fraction } & \multicolumn{3}{|c|}{ Predicted weight fraction } & \multirow{2}{*}{$\begin{array}{l}\text { Average absolute } \\
\text { prediction error }\end{array}$} \\
\hline & Basil & Cinnamon & Garlic & Basil & Cinnamon & Garlic & \\
\hline $1 \mathrm{~T}$ & 0.218 & 0.015 & 0.767 & 0.192 & 0.129 & 0.679 & 0.076 \\
\hline $4 \mathrm{~T}$ & 0.413 & 0.366 & 0.221 & 0.323 & 0.418 & 0.258 & 0.06 \\
\hline \multirow[t]{2}{*}{$5 \mathrm{~T}$} & 0.052 & 0.777 & 0.171 & 0.052 & 0.839 & 0.109 & 0.041 \\
\hline & & & & & \multicolumn{3}{|c|}{ Mean Square Error $(\mathrm{MSE})=0.0035$} \\
\hline
\end{tabular}

the PCA-MLP structure over MLP. It should be noted that in general, the PCA-MLP structure does not necessarily perform better than the MLP because PCA may not preserve all the discrimination information of the original data. In our case, the performance of PCAMLP indicates that most of the discrimination information of the original data was preserved. It was not surprising that TDNN provided the smallest prediction MSE because it can capture the additional information about time-related differences in sensors' responses. In this study, the increase in the number of independent parameters was outweighed by more reliable information captured from the transient response of the sensors, which was not available in the instantaneous data used in MLP and PCA-MLP.

This improvement of TDNN over MLP had also been more significant in a reported application for e-noses' pattern recognition (Zhang and others 2003).

Figure 5 shows the distribution of the prediction points, including those from MLP, PCA-MPL, and TDNN, in the simplex space. It should be noted that the predictions from all 3 methods were skewed toward cinnamon compared with the experimental spice mixture points. In other words, all these methods tended to predict higher cinnamon fractions within a mixture. The intensity of cinnamon smell is the strongest among the 3 spices, and the volatile concentration of cinnamon is 10-fold that of basil and garlic volatiles in the volatile extracts from the spice mixture (mix ratio 1:1:1) (Zhang 2003a). Additionally, the sensors of the Neotronics e-nose used in the study are made with polypyrrole resins (Marsili 1995), which have stronger interactions with molecules containing carbonyl such as cinnamaldehyde and cinnamyl acetate compared with the oxygen-containing compounds in the basil and sulfur-containing compounds in garlic (Pine 1987). Cinnamaldehyde and cinnamyl acetate account for more than $90 \%$ of total volatiles in the cinnamon (Zhang and others 2005). This skewed prediction can be due to the following possibilities: the e-nose sensors might be overwhelmed by the cinnamon volatiles during the "sniffing" process of e-nose operations, or there might be cinnamon odor residues in the sampling or sensor chamber despite the e-nose cleaning procedure.

To predict unknown mixture compositions, the training points for prediction model building cover the whole mixture space and should be evenly distributed within that space. It also means that the model developed based on the training data cannot be used to predict an unknown mixture whose compositions are not within the training mixture space. Evenly distributed training points ensure a fair estimation of the parameters of the prediction model.

There are 2 main factors that influence how many training points will be necessary for predictive model building: the number of components being mixed, and the complexity of the sensors' response to the mixture. The more components being mixed and the more complex the sensors' response, the more training points will be necessary. The number of samples necessary for each training point depends on the variability of the e-nose response to an identical mixture composition, the differences among the mixtures with different compositions, and the target prediction accuracy of the mixture compositions.

For a ternary mixture system, the prediction of compositions is actually a 2-dimensional problem due to the built-in constraint of compositional data. Ideally, if the e-nose response data show a data structure that the 1st 2 principal components explain a large proportion of total variance yet the 2 nd principal component accounts for a signif-

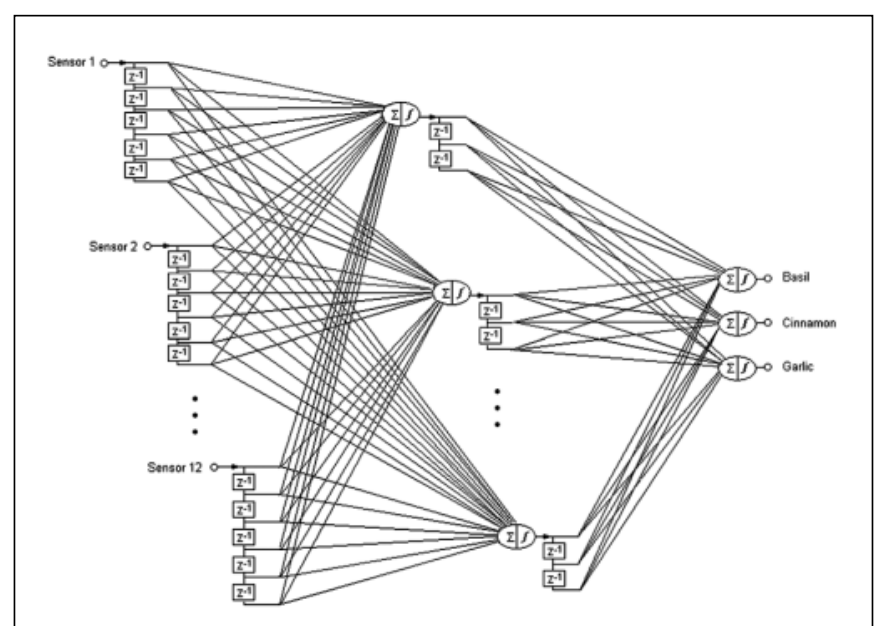

Figure 4-The optimal topology of time-delay neural network (TDNN)

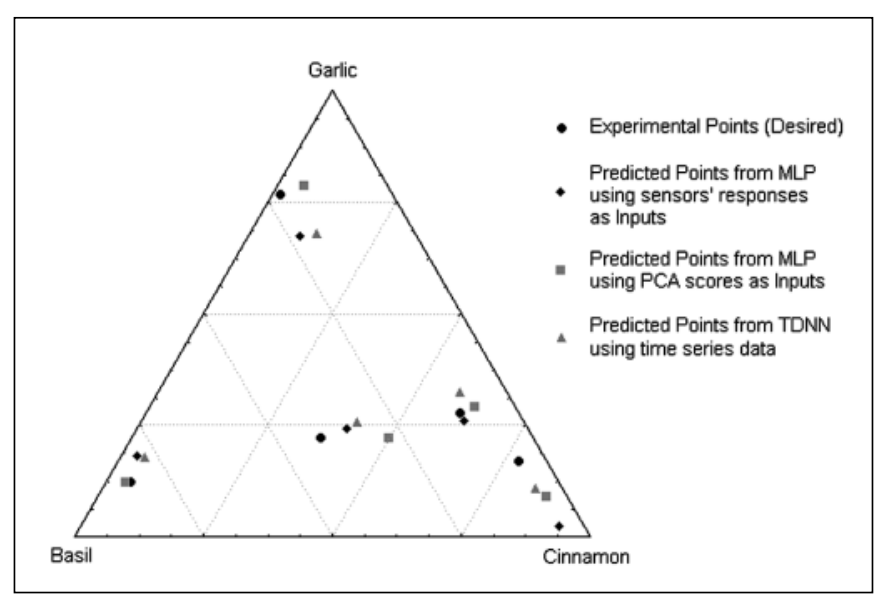

Figure 5-The distribution of the prediction points, including those from e-nose multilayer perceptron (MLP), principal component analysis-multilayer perceptron PCA-MLP and time-delay neural network (TDNN) analysis, in the simplex space 
icant proportion of total variance, a better composition prediction may result for the PCA-MLP method. Accordingly, if a mixture with 4 components is under investigation, the 1st 3 principal components are expected to explain a large proportion of total variance, yet the 3rd principal component should still account for a significant proportion of total variance. The e-nose response data obtained in this study did not have these ideal properties. Among the selected 1st 2 principal components used in PCA-MLP, the 1st principal component explained more than $98 \%$ of total variance, resulting in the 2nd principal component having a relatively high noise-to-signal ratio. The ideal structure of the data may not only improve the prediction ability of the PCAMLP structure, but also other prediction models used, which include both the multivariate statistical and neural network methods. A proper sensor array design/selection may make the sensors' responses approach ideal conditions.

It should be noted that neural networks have the advantage over the traditional statistical methods only when the investigated data structures are highly complex. In case the investigated data structures are simple, alternative statistical methods such as principal component regression or partial least squares may perform better than neural networks in a quantitative e-nose study.

\section{Conclusions}

$\mathrm{T}$ his study showed that it is feasible to quickly predict the compositions of mixtures that differ in their olfactory attributes using e-nose technology. The developed experimental method (experimental design/data collection) provided well-structured and sufficient data to perform further analysis. The 3 neural network models-MLP, PCA-MLP, and TDNN-worked well as ingredient percentage prediction methods for e-nose applications, with similar accuracy. In this study, PCA-MLP performed marginally better than MLP, and TDNN performed marginally better than PCA-MLP. How well the relative accuracy and efficiency of the developed methods (e-nose and neural networks) compared with the traditional methods of odor analysis (sensory and GC) will be presented in part II of this study.

\section{Acknowledgments}

Florida Agricultural Experiment Station Journal Series Nr R-10785.

\section{References}

Acree T, Arn H. 2004. FlavorNet. Cornell University, Geneva, N.Y. Available from: http://www.nysaes.cornell.edu/flavornet/chem.html. Accessed 2002 June.

Adams RP. 1995. Identification of essential oil components by gas chromatography/mass spectroscopy. Carol Stream, Ill.: Allured Publishing. 469 p.

Alpha-M.O.S. 1997. Study of pure hops and blends with an electronic nose. Hillsborough, N.J.: Alpha MOS, Inc. Application note 24 of Alpha-M.O.S.

Cornell JA. 1990. Experiments with mixtures-designs, models, and the analysis of mixture data. 2nd ed. New York: John Wiley \& Sons. 656 p.

Dittmannn B, Zimmermann B, Engelen C, Jany G, Nitz S. 2000. Use of MS-Sensor to discriminate between different dosages of garlic flavoring in tomato sauce. J Agric Food Chem 48:2887-92.

Garbe D, Surburg H, Beuer K. 2001. Common fragrance and flavor materials: preparation, properties and uses. 4th ed. New York: John Wiley and Sons. 293 p.

Gardner JW, Bartlett PN. 1999. Electronic noses principles and applications. Oxford, U.K.: Oxford Univ. 264 p.

Harper WJ. 2001. The strengths and weaknesses of the electronic nose. In: Rouseff RL, Cadwallader KR, editor. Headspace analysis of foods and flavors-theory and practice. New York: Kluwer Academic. p 59-71.

Haykin S. 1999. Neural networks-a comprehensive foundation. 2nd ed. Upper Saddle River, N.J.: Prentice-Hall. 842 p.

Khattree R, Naik DN. 2000. Multivariate data reduction and discrimination with SAS software. Cary, N.C.: SAS Inst. 584 p.

Marsili R. 1995. The electronic nose. Northbrook, Ill.: Weeks Publishing Co. Available from: http://www.foodproduct design.com/archive/1995/0695QA.html. Accessed 2002 June.

Pine SH. 1987. Organic chemistry. 5th ed. New York: McGraw-Hill. p 1187.

Principe JC, Euliano NR, Lefebvre WC. 1999. Neural and adaptive systems: fundamentals through simulations. New York: John Wiley \& Sons. 672 p.

Uhl SR. 2000. Spices, seasonings and flavorings. Lancaster, Pa.: Technomic Publishing. $329 \mathrm{p}$.

Zhang H. 2003a. Predicting spice mixture composition: comparing electronic nose, gas chromatography, and sensory methods [DPhil Dissertation]. Gainesville, Fla.: Univ. of Florida. 124 p. Available from: Univ. of Florida Library, Gainesville, FL: LD1780 2003.Z632.

Zhang H. 2003b. The use of electronic noses in evaluating food quality. In: Tothill I, editor. Rapid and on-line instrumentation for food quality assurance. Cambridge, U.K.: Woodhead. p 324-38.

Zhang H, Balaban MO, Principe JC. 2003. Improving pattern recognition of electronic nose data with time-delay neural networks. Sensors Actuators B: Chem 96(1/2):385-9.

Zhang H, Balaban MO, Principe JC, Portier K. 2005. Quantification of spice mixture compositions by electronic nose: Part II. Comparison with GC and sensory methods. J Food Sci 70(4):E259-264. 